\title{
Correlative and Multiplexed Microscopy for 2-D Chalcogenide Semiconductors
}

\author{
Jeffrey D. Cain ${ }^{1}$, Eve D. Hanson ${ }^{1}$ and Vinayak P. Dravid ${ }^{1}$ \\ 1. Department of Materials Science and Engineering, and the NUANCE Center, Northwestern University, \\ Evanston, Illinois USA
}

The science and engineering community has been rapidly exploring the landscape of two-dimensional materials, particularly in their single- or few layer forms [1]. Dominating this landscape are the layered chalcogenides [2] which are diverse in chemistry, structure and properties; with over 100 primary members of this materials family already being reported. Driven by quantum confinement single layers (or few, in some cases) of these materials exhibit electronic, optical, and transport properties that diverge dramatically from their bulk counterparts. The field has evolved considerably since the time when single or few layer flakes were "synthesized" by the scotch-tape mechanical cleavage method. New more sophisticated methods for controlled synthesis (or thinning), deposition and chemical exfoliation have been developed that can "dial" the number of layers with large areal coverage on diverse substrates. Further, the 2D chalcogenide layers are being used as "substrates" onto which other dimensionally confined structures are being integrated in the spirit of nanoscale composites. Some composite structures exhibit synergy of multiple functionalities of the individual components while in some cases they represent quantum coupling or unusual behavior that is contrary to nominal synergy or proportional contribution of individual components.

Thus, the 2-D chalcogenide systems represent remarkably diverse semiconductors with ample opportunities for innovative geometries and complex architectures. The 2-D chalcogenides are clearly following the logical evolution of classical semiconductors and their heterostructure or multilayer forms. However, characterization of 2-D chalcogenides represents particular challenges because the potential fragility of single (or few) molecular thickness, and rather dramatic changes in electronic structure that often result from just a minute changes in number of layers or substrate confinement or dangling bonds, to name a few. The characterization needs for 2-D chalcogenides range from rapid through-put imaging [3] to detailed analysis of (spatially varying) physical, chemical or electronic attributes [4]. It involves basic atomic force microscopy (AFM) to validate molecular-level thickness of deposited layers or piezoelectric behavior, while HAADF/STEM atomic-scale imaging, EELS/EDS spectroscopy and spectroscopic imaging are essential for vertical/in-plane heterostructures and alloys. Raman spectroscopy/imaging, photoluminescence (PL), second-harmonic generation (SHG) imaging are some of the typical optical techniques often used to decipher some electronic aspects of 2-D chalcogenides. Thus, correlative and multiplexed microscopy or characterization is essential in 2-D chalcogenide field, especially to probe the nucleation and growth mechanisms in vapor-based growth approaches. For example, we have shown that a "buckyball"-type nucleating agent comprising Mo-S-O complexes may be precursor to single layer growth of $\mathrm{MoS}_{2}$. Fig. 1 shows chemical mapping of the buckyball of Mo-S$\mathrm{O}$, which results in lateral growth of single-layer, shown schematically in Fig. 2. The presentation will cover correlative and multiplexed microscopy approach for 2-D chalcogenide semiconductors, especially vertical heterostructures $\left(\mathrm{V}_{\mathrm{H}}\right)$, radial heterostructures $\left(\mathrm{R}_{\mathrm{H}}\right)$ and alloy/doped systems. [5]

\section{References:}

[1] Novoselov, K.S., et al., Science, 2004. 306(5696): p. 666-669. 
[2] Mak, K.F., et al., Phys Rev Lett, 2010. 105(13): p. 136805.

[3] Late, D.J., et al., Advanced Functional Materials, 2012. 22(9): p. 1894-1905.

[4] Huang, Y.-K., et al., ACS Nano, 2014. 8(10): p. 10851-10857.

[5] This work is partly supported by NSF-DMR and AFOSR programs.

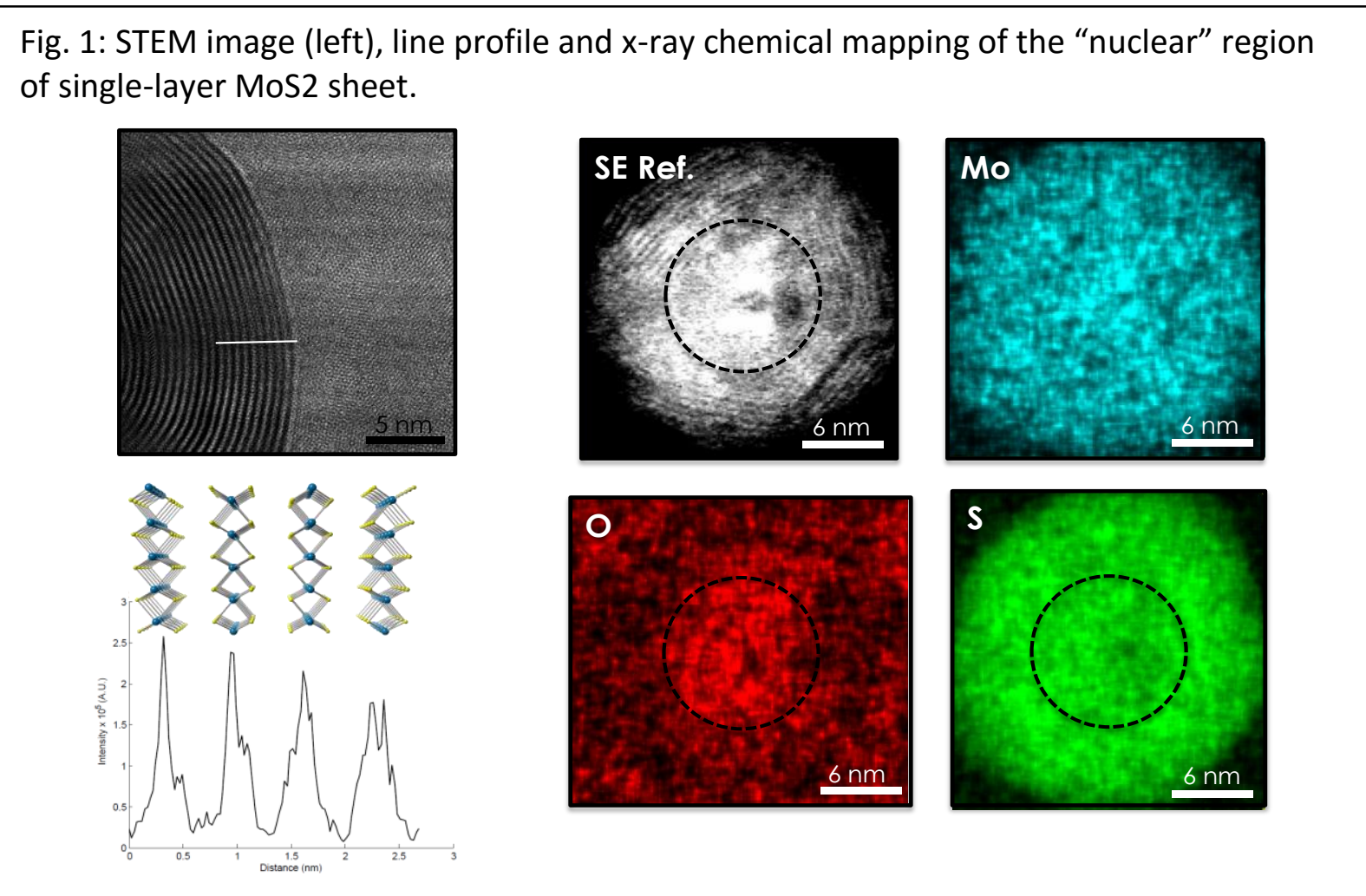

Fig. 2: Schematic illustration of growth mechanism of 2-D MoS2 sheet, based on Mo-O-S nucleus complex in the center of the sheet.

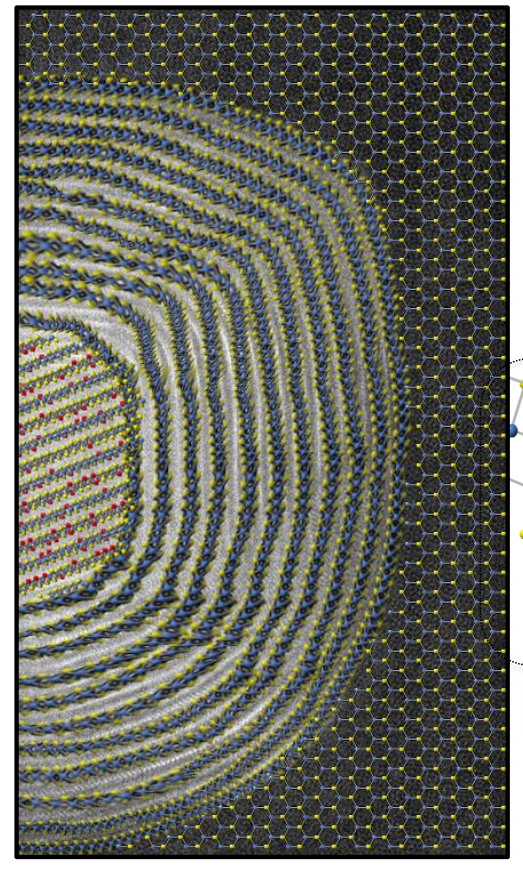

"Self-seeding" growth via oxy-sulfide core 